\section{Lymphoid Necrosis Induced by Lipid Emulsions}

LYMPHOID tissue exposed to certain lipid emulsions undergoes a gross and widespread necrosis, while exposure either to the lipid or emulsifier alone produces only minor 'reactive' changes. This phenomenon, which, so far as we know, had not been previously reported, was first described by one Bennett and Shivas ${ }^{1}$. Further work still in progress has shown that necrosis can be produced by a wide variety of lipid emulsions and the effect appears to be a general property of these. Emulsions containing 20 per cent w/v ethyl stearate, ethyl laurate, ' $F$ 99' (a preparation of ethyl esters of linoleic and linolenic acids), stearic acid, lauric acid, olive oil and liquid paraffin have been given in a single dose of $2 \mathrm{ml}$. to fully grown young rats by the intraperitoneal route under direct vision at laparotomy with ether anæsthesia. The emulsifiers used are 'Tween 80', ox bile and gum acacia. Glyceryl monostearate emulsions were prepared without any added emulsifying agent. Unemulsified lipids and aqueous solutions of emulsifying agents (with the exception of ox bile) have also been administered separately at concentrations equivalent to those in the emulsions.

Operative mortality is negligible and the animals, which are killed at intervals of 1-8 days after injection, are no more indisposed than might be expected from the anæsthesia and trauma. Necrosis found in the slightly enlarged mediastinal lymph nodes which constitute the regional drainage varies in extent and severity with the individual emulsion, being most severe with ethyl stearate (Figs. 1 and 2). The maximum effect is attained about 5 days after injection. 'Reactive' changes resulting from the lipids or emulsifiers given alone consist chiefly of a dilatation of the sinuses which become filled with large mononuclears looking like macrophages (Fig. 3). In both necrotic and non-necrotic lymph nodes a very interesting feature is the alteration in morphology of surviving cells reminiscent of that described by the Gillmans ${ }^{2}$ in their work with trypan blue and of the 'hepatic microgranulomas' in dogs given intravenous fat emulsion reported by Meyer et $a l .^{3}$
After severe necrosis (Fig. 2) regeneration seems to proceed from fibrocellular elements which pre. sumably escape necrosis. The necrotizing action thus appears selective and there have been no adhesions in the peritoneal cavity or evidence of damage other than to lymphoid tissue.

These findings pose many questions which cannot yet be answered. Nevertheless, it seems reasonable to conclude that the action of the emulsions in producing necrosis is to a large extent independent of the nature of the lipid used. Occurring with free and esterified fatty acids both saturated and unsaturated, with the hydrocarbon oil liquid paraffin and with the 'essential' fatty acids, it would seem to depend primarily on their physical state. Although the actual mechanism is quite obscure and much work remains to be done, the lipid emulsions may well provide a new research tool for investigation of the reticuloendothelial system and it is with this hope that we venture, by this interim report, to bring it to the notice of workers in all fields of biological research.

A. A. Shivas

Department of Pathology,

G. P. Fraser

University of Edinburgh.

1 Bennett, H. S., and Shivas, A. A., J. Fac. Radiol., 5, 261 (1954).

2 Gillman, J., and Gíllman, T., Clin. Proc. 8, 222 (1949)

${ }^{3}$ Meyer, C. E., Fancher, J. A., Schurr, P. E., and Webster, H. D.,

\section{RADIOBIOLOGY}

\section{Concentration of Cæsium-137 in the Coastal Waters of Japan (1959)}

I HAVE analysed bittern and carnallite of industrial origin and deduced the concentration of cæsium-137 in the coastal waters in early 1958 of Japan as

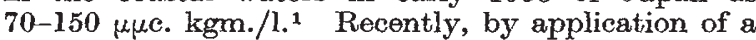
low-level $\beta$-counting equipment, cæsium-137 has been successfully determined by direct treatment of 6-20 litres of sea-water.

Separation of $20 \mathrm{mgm}$. of carrier cæsium which had been originally added to the sample water was
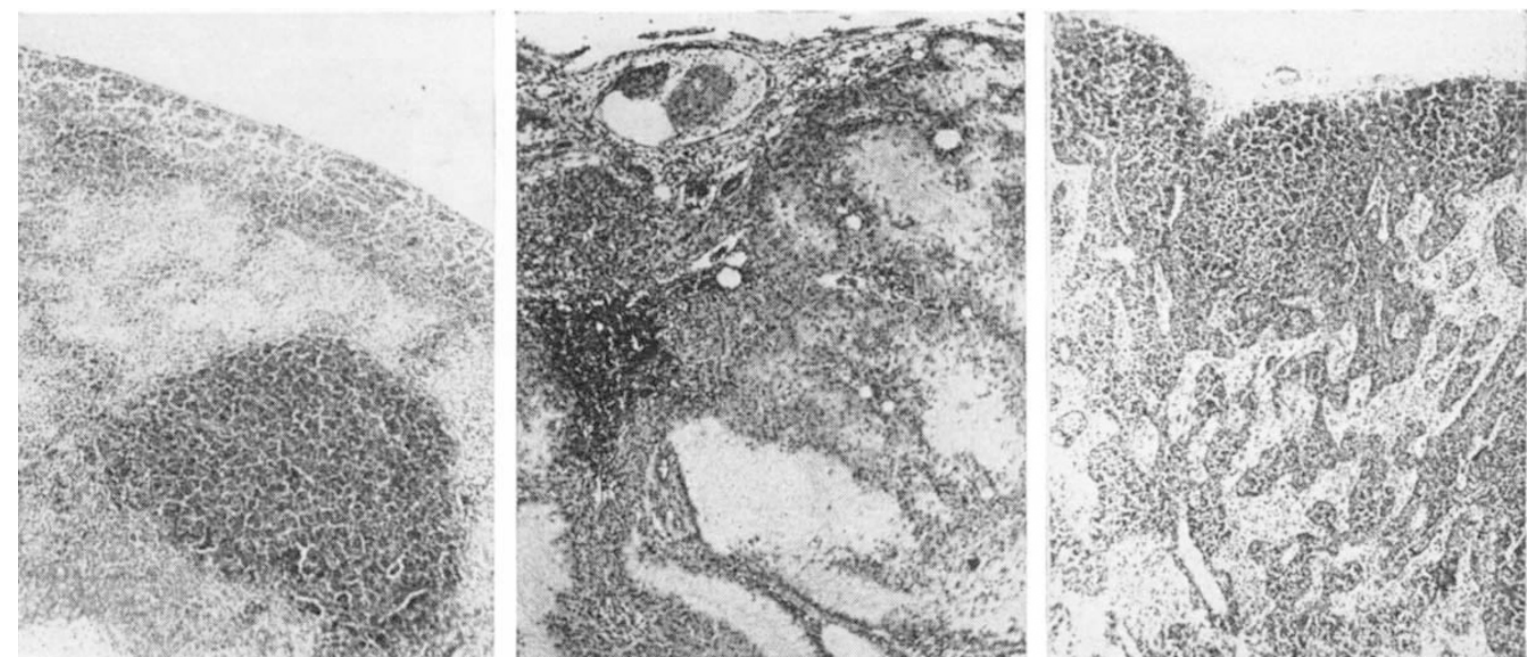

Fig. 1 (left). Early necrosis in a mediastina fymph node 1 day after intraperitoneal injection of an emulsion of ethyl stearate. The darkly-staining areas are surviving normal lymphoid tissue (H. and $\mathrm{E}$. $\times c .60$ ). Fig. 2 (centre). Large tracts of amorphous necrotic debris six days after injection of the emulsion. Note the proliferation of flbrocellular tissue and the small area of surviving lymphoid tissue (left of centre) (H. and E. $\times c .50)$. Fig. 3 (right). Mediastinal lymph node 8 days after intraperitoneal injection of olive oil.
Note the dilated sinuses crowded with mononuclears of macrophagic appearance, and the absence of necrosis $(H$, and $\mathrm{E}$. $\times c$. 60 ) 